typeset using JPSJ.sty $<$ ver.1.0b $>$

\title{
Numerical Study of Aging in the Generalized Random Energy Model
}

\author{
Munetaka SASAKI and Koji Nemoto \\ Division of Physics, Hokkaido University, Sapporo 060-0810
}

(Received )

\begin{abstract}
Magnetizations are introduced to the Generalized Random Energy Model (GREM) and numerical simulations on ac susceptibility is made for direct comparison with experiments in glassy materials. Prominent dynamical natures of spin glasses, i.e., memory effect and reinitialization, are reproduced well in the GREM. The existence of many layers causing continuous transitions is very important for the two natures. Results of experiments in other glassy materials such as polymers, supercooled glycerol and orientational glasses, which are contrast to those in spin glasses, are interpreted well by the Single-layer Random Energy Model.
\end{abstract}

KEYWORDS: aging, generalized random energy model, memory effect, reinitialization, temperature specific dynamics, cumulative dynamics

\section{$\S 1 . \quad$ Introduction}

In spin glasses, it is well known that dynamical behavior strongly depends on history of the system after quenching from above the transition temperature $T_{\mathrm{c}}$. These phenomena are called aging and have been studied with various experimental protocols such as the isothermal 1, 目) the $T$-shift国 and the $T$-cycle one. been studied along two different pictures, i.e., so-called droplet picture19, (1), 12, (13) and hierarchical picture.0.78) The Generalized Random Energy Model (GREM)14,15, 16,17) is a model that belongs to the latter. This model has a hierarchical structure causing continuous transitions as the system is cooled down. These continuous transitions correspond to successive branching process of free energy in the hierarchical picture. Bouchaud and Dean 14 have shown that aging naturally occurs in this model and the time correlation function $C\left(t+t_{\mathrm{w}}, t_{\mathrm{w}}\right)$ satisfies a $t / t_{\mathrm{w}}$ scaling law. Furthermore, we have recently made simulation 18.19 ) similar to experiments on aging phenomena with temperature variations. As the consequence, it has been shown that results of experiments are reproduced well according to the hierarchical picture.

Recently, Jonason et at20 have made a new experiment, in which curious dynamical natures of spin glasses are observed very clearly. This experiment consists of the following two runs. In the first run, the sample is continuously cooled from $T_{\max }\left(>T_{\mathrm{c}}\right)$ to $T_{\min }\left(<T_{\mathrm{c}}\right)$ at a constant rate, 
and is immediately reheated at the same rate. During the cooling and the reheating, out-of-phase ac-susceptibility $\chi^{\prime \prime}$ is measured as a function of temperature. We call this curve as $\chi_{\text {ref }}^{\prime \prime}$. The difference between the observed value in the cooling and that in the reheating is not observed in spin glasses, while the hysteresis is observed in other glassy materials, such as polymer glasses,21) orientational glasses22) and disordered ferromagnets 23 . In the second run, the sample is cooled from $T_{\max }$ to a waiting temperature $T_{\text {wait }}\left(T_{\min }<T_{\text {wait }}<T_{\mathrm{c}}\right)$, and is kept at $T_{\text {wait }}$ during a certain time interval. The sample ages and $\chi^{\prime \prime}$ decreases during the interval. Then the system is cooled to $T_{\min }$ and is reheated to $T_{\max }$ without any stops. Hereafter this curve is denoted as $\chi_{\text {wait }}^{\prime \prime}$.

An important result of this experiment is that $\chi_{\text {wait }}^{\prime \prime}$ merges with $\chi_{\text {ref }}^{\prime \prime}$ at very beginning of the resumed cooling as if the system forgets the aging at $T_{\text {wait }}$ (reinitialization of aging). But this aging is still imprinted and a dip of $\chi_{\text {wait }}^{\prime \prime}$ created in the cooling stage is exactly recovered in the

reheating stage (memory effect). As Hammann et al have pointed out,24 these two effects make dynamics in spin glasses temperature specific in the sense that the effect of aging at $T_{\text {wait }}$ (or the difference between $\chi_{\text {ref }}^{\prime \prime}$ and $\chi_{\text {wait }}^{\prime \prime}$ ) only appears near $T_{\text {wait }}$ in the both cooling and reheating stages. The purpose of this manuscript is to study aging phenomena of the GREM with this protocol.

The organization of this manuscript is as follows: In $\S 2$, the GREM is explained and magnetizations are introduced to this model. In $\S 3$, the results of the simulations are presented. In $\S 4$, it is discussed how a variety of aging phenomena observed in glassy materials are interpreted within the GREM.

\section{§2. $\quad$ Model}

The GREM is schematically shown in Fig. 1. This model consists of $L$ layers which are piled up hierarchically. The bottom points represent accessible states of the system and each branch represents a barrier over which the system goes to another state. Each branching point has $N$ branches, so that the number of states is $N^{L}$. It is assumed that $N$ is large enough. Energy barriers of the $n$-th layer counted from the bottom, $E_{n}$, are given randomly and independently according to the distribution

$$
\rho_{n}\left(E_{n}\right)=\frac{1}{T_{\mathrm{c}}(n)} \exp \left[-\frac{E_{n}}{T_{\mathrm{c}}(n)}\right],
$$

where $T_{\mathrm{c}}(n)$ is the transition temperature of the $n$-th layer. From the distribution, the averaged relaxation time of the $n$-th layer $\langle\tau(n)\rangle$ is easily calculated as

$$
\begin{aligned}
\langle\tau(n)\rangle & \equiv \int_{0}^{\infty} \mathrm{d} E_{n} \rho\left(E_{n}\right) \tau_{0} \exp \left(E_{n} / T\right) \\
& =\left\{\begin{array}{cc}
\frac{T \tau_{0}}{T-T_{\mathrm{c}}(n)} & \left(T>T_{\mathrm{c}}(n)\right), \\
\infty & \left(T \leq T_{\mathrm{c}}(n)\right),
\end{array}\right.
\end{aligned}
$$

where $\tau_{0}$ is a microscopic time scale. This means that a transition from the ergodic phase to the non-ergodic phase occurs at $T_{\mathrm{c}}(n)$ in the $n$-th layer. The transition temperatures are chosen so as 
to satisfy the inequality $T_{\mathrm{c}}(1)<T_{\mathrm{c}}(2)<\cdots<T_{\mathrm{c}}(L)$. Therefore, transitions occur continuously from the uppermost (the $L$-th) layer to the lowest one.

Next, let us explain how magnetizations are introduced to the GREM.18, 19) It is natural to assume that the nearer two states $\alpha$ and $\beta$ are the stronger the correlation between the two magnetizations is, and that the distance between the two states $d(\alpha, \beta)$ is measured by the layer from which they are separated, e.g., in Fig. $1 d(\alpha, \beta)=1$ and $d(\alpha, \gamma)=2$. To incorporate these aspects, we assign the value of the magnetization $M_{\alpha}$ to state $\alpha$ as

$$
M_{\alpha}=\mathcal{M}_{0}(\alpha)+\mathcal{M}_{1}\left(\alpha_{1}\right)+\cdots+\mathcal{M}_{L-1}\left(\alpha_{L-1}\right)
$$

where $\alpha_{k}$ is the $k$-th ancestor of $\alpha$ and $\mathcal{M}_{k}\left(\alpha_{k}\right)$ is a contribution from the branching point. The value of $\mathcal{M}_{k}\left(\alpha_{k}\right)$ is given independently and randomly from distribution $D_{k}\left(\mathcal{M}_{k}\right)$ with the mean value $\overline{\mathcal{M}_{k}}=0$. If $d(\alpha, \beta)=k$, the correlation between $M_{\alpha}$ and $M_{\beta}$ comes from the common contributions of $\mathcal{M}_{n}(n=k, k+1, \cdots)$ to these magnetizations

$$
\overline{M_{\alpha} M_{\beta}}=\sum_{n=k}^{L-1} \overline{\mathcal{M}_{n}^{2}}
$$

It decreases monotonically as $k$ increases and the barrier between two states becomes higher, which is observed in the SK model.25) In the present simulation, the same uniform distribution is chosen for $\mathcal{M}$ of all the layers as

$$
D_{n}\left(\mathcal{M}_{n}\right)=\left\{\begin{array}{cc}
\frac{\sqrt{L}}{2} & \left(\left|\mathcal{M}_{n}\right| \leq \frac{1}{\sqrt{L}}\right) \\
0 & \left(\left|\mathcal{M}_{n}\right|>\frac{1}{\sqrt{L}}\right)
\end{array}\right.
$$

The range of the distribution is chosen so that the variance of $M_{\alpha}$ is independent of $L$.

The dynamics of the system is described by the master equation for the probability $P_{\alpha}(t)$ of finding the system at a state $\alpha$ at time $t$,

$$
\frac{\mathrm{d}}{\mathrm{d} t} P_{\alpha}(t)=\sum_{\beta \neq \alpha} W_{\alpha \beta}(t) P_{\beta}(t)-\sum_{\beta \neq \alpha} W_{\beta \alpha}(t) P_{\alpha}(t),
$$

where $W_{\alpha \beta}(t)$ is the transition rate at $t$ for going from $\beta$ to $\alpha$ in a unit time. The uniform distribution is chosen for the initial condition, i.e., $P_{\alpha}(0)=N^{-L}$ for each $\alpha$. This means that the system is quenched from an infinitely high temperature. The transition rate $W_{\alpha \beta}(t)$ is given as

$$
W_{\alpha \beta}(t)=\tau_{0}^{-1} \sum_{k=d(\alpha, \beta)}^{L} \frac{1}{N^{k}}\left\{\exp \left[-\frac{\sum_{n=1}^{k} E_{n}(\beta)+H(t) M_{\beta}}{T(t)}\right]-\exp \left[-\frac{\sum_{n=1}^{k+1} E_{n}(\beta)+H(t) M_{\beta}}{T(t)}\right]\right\},
$$

where $H(t)$ is magnetic field and $E_{L+1}$ is hypothetical energy whose value is infinity. The factor in the braces on the right hand represents the probability that the system can be activated to the $k$-th layer but not to the $k+1$-th layer, and the factor $1 / N^{k}$ represents the probability that the system falls into $\alpha$. The details of how the dynamics is simulated in the limit $N \rightarrow \infty$ are presented in ref. 18 . 


\section{§3. Results of Simulations}

The simulation is done in the GREM with $L=2, T_{\mathrm{c}}(1)=0.5$ and $T_{\mathrm{c}}(2)=1.0$. The amplitude and the period of the applying ac-field are 0.1 and $100 \tau_{0}$, respectively. The system is cooled at the rate of $2.0 \times 10^{-5} \mathrm{~T} / \tau_{0}$, and is immediately reheated at the same rate in the case of $\chi_{\text {ref }}^{\prime \prime}$ measurement. The cooling is intermitted at $T_{\text {wait }}=0.7$ for $1.0 \times 10^{5} \tau_{0}$ when $\chi_{\text {wait }}^{\prime \prime}$ is measured. The waiting time is comparable to the sweeping time from $T_{\max }$ to $T_{\min }$. The temperature $T_{\text {wait }}$ is set so as to satisfy

$$
T_{\mathrm{c}}(1)<T_{\text {wait }}<T_{\mathrm{c}}(2) \text {. }
$$

In Fig. 2 there is obvious hysteresis, which does not disappear even in the case of slower rates (to $2.0 \times 10^{-6} \mathrm{~T} / \tau_{0}$ ) and longer periods (to $10^{3} \tau_{0}$ ). An important result of the simulation is reinitialization, i.e., $\chi_{\text {wait }}^{\prime \prime}$ merges with $\chi_{\text {ref }}^{\prime \prime}$ in the early stage of the resumed cooling.

In order to visualize the effect of the aging at $T_{\text {wait }}$ more clearly, the difference between $\chi_{\text {ref }}^{\prime \prime}$ and $\chi_{\text {wait }}^{\prime \prime}$ is shown in Fig. 3. If we regard the difference as a measure of the aging effect, we notice that the system behaves as if it quickly forgets the aging in the cooling stage (reinitialization) and again remembers when the system is heated back near $T_{\text {wait }}$ (memory effect), which implies that dynamics of the GREM is temperature specific.

To reveal the mechanism of these effects, $\chi_{0}^{\prime \prime}$ and $\chi_{1}^{\prime \prime}$ evaluated from $\mathcal{M}_{0}$ and $\mathcal{M}_{1}\left(\chi^{\prime \prime}=\chi_{0}^{\prime \prime}+\chi_{1}^{\prime \prime}\right)$ are plotted in Fig. 4. The rapid increase of $\chi^{\prime \prime}$ just after the resumed cooling is brought by $\chi_{0}^{\prime \prime}$. As for the relaxation at $T_{\text {wait }}$, the first layer is quickly equilibrated and $\chi_{0}^{\prime \prime}$ almost retains a constant value because $T_{\text {wait }}>T_{\mathrm{c}}(1)$, while $\chi_{1}^{\prime \prime}$ decreases since $T_{\text {wait }}<T_{\mathrm{c}}(2)$. In this sense, $\chi_{1}^{\prime \prime}$ is the affected part and $\chi_{0}^{\prime \prime}$ is the unaffected one. As the resumed cooling goes on, the affected part decreases and the unaffected part increases because the peak of $\chi_{0}^{\prime \prime}$ and that of $\chi_{1}^{\prime \prime}$ are near $T_{\mathrm{c}}(1)$ and $T_{\mathrm{c}}(2)$ respectively. As the result, $\chi_{\text {wait }}^{\prime \prime}$ merges with $\chi_{\text {ref }}^{\prime \prime}$ as if the system forgets the aging at $T_{\text {wait }}$. But $\chi_{1}^{\prime \prime}$ again recovers a large contribution to $\chi^{\prime \prime}$ and the system remembers the aging when the system is reheated to $T_{\text {wait }}$.

\section{$\S 4 . \quad$ Discussion}

As Hammann et al have pointed out,24) dynamics in glassy materials seems to be classified into two distinct types. In the first type, memory effect and reinitialization, which make the dynamics temperature specific, are observed. Spin glasses belong to this type as mentioned in 8 囵. In the other type, memory effect exists but reinitialization does not exist. Since the system is never reinitialized after quenching from above $T_{\mathrm{c}}$, the dynamics is cumulative. In this dynamics, the time evolution in the vicinity of $T_{\mathrm{c}}$ is very important. Polymers, 21, 26 supercooled glycerol 27) and the orientational glasses 22, 28) belong to this type.

Now let us discuss how these two distinct types of dynamics observed in glassy materials are interpreted within the GREM. Time evolution of energy distribution $P_{n}\left(E_{n}, t\right)$, which is defined as the probability density that the system is found at time $t$ in one of the states whose energy of the $n$-th 
layer is $E_{n}$, is very different according as $T>T_{\mathrm{c}}(n)$ or $T<T_{\mathrm{c}}(n)$. 1 ) In both cases, $P_{n}\left(E_{n}, t\right)$ tries to approach the equilibrium distribution proportional to $\exp \left(E_{n} / T\right) \rho_{n}\left(E_{n}\right)=\exp \left(\left\{\frac{1}{T}-\frac{1}{T_{\mathrm{c}}(n)}\right\} E_{n}\right)$ with increasing time. But the sign of the exponent is different in the two cases. Consequently, $P_{n}\left(E_{n}, t\right)$ quickly converges to the equilibrium distribution $P_{n}^{\mathrm{eq}}\left(E_{n}\right)=\left\{\frac{1}{T}-\frac{1}{T_{\mathrm{c}}(n)}\right\} \exp \left(\left\{\frac{1}{T}-\frac{1}{T_{\mathrm{c}}(n)}\right\} E_{n}\right)$ if $T>T_{\mathrm{c}}(n)$, whereas $P_{n}\left(E_{n}, t\right)$ has a peak moving to higher energy level with increasing time if $T<T_{\mathrm{c}}(n)$ (time evolution of $P_{n}\left(E_{n}, t\right)$ in the case of $T<T_{\mathrm{c}}(n)$ is shown in Fig. 6, which is mentioned more closely later on). In this model, the peak position indicates the age of the layer. This means that the age of layers which satisfy $T>T_{\mathrm{c}}(n)$ is kept to be 0 since the peak of $P_{n}^{\mathrm{eq}}\left(E_{n}\right)$ is located at $E_{n}=0$.

Although the aging process in each layer is cumulative, the dynamics of the GREM is temperature specific. Now let us discuss what happens in the case of $L \gg 1$. For a given temperature $T<T_{\mathrm{c}}(L)$, there exists the $n$-th layer which satisfies $T_{\mathrm{c}}(n-1)<T<T_{\mathrm{c}}(n)$. As discussed in ref. 18, the crucial point is the fact that the layers below $n$ are quickly equilibrated and do not contribute to slow dynamics, while those above $n$ are almost quenched and they behave as if the time evolution stops. This means that the $n$-th layer is the activated one and mainly dominates slow dynamics of the system. This activated layer changes with temperature, that causes temperature specific dynamics of the GREM. Memory of aging at a given temperature is stored in the corresponding activated layer as a peak position of $P_{n}\left(E_{n}, t\right)$. The memory is preserved while the system is cooled since the layer becomes frozen, and is reinitialized if the system is heated up to a certain temperature $T+\Delta T>T_{\mathrm{c}}(n)$ because the peak of $P_{n}\left(E_{n}, t\right)$ created at $T$ is destroyed.

In the Single-layer Random Energy Model (GREM with $L=1$ ), the dynamics is cumulative because changes of the activated layer do not occur. In Fig. 5, the relaxation of $\chi^{\prime \prime}$ during positive $T$-cycle in this model is shown. The relaxation highly proceeds while the system is heated up, which is usually observed in cumulative systems.26) On the other hand, relaxation of $\chi^{\prime \prime}$ is reinitialized by positive $T$-cycle in the GREM.18)

This understanding of aging phenomena observed in glassy materials is just the same as that obtained from studies of mean-field spin glasses, 29 in which it has been concluded that dynamics of mean-field models with a one-step replica symmetry breaking solution is cumulative and that with a full replica symmetry breaking solution is temperature specific.

In order to see how activated layers and frozen ones age, an example of time evolution of $P_{n}\left(E_{n}, t\right)$ is shown in Fig. 6. After quenching from an infinitely high temperature, the system is kept at $T=0.45$. The number of layers is three and the transition temperatures are set at $T_{\mathrm{c}}(1)=0.6$, $T_{\mathrm{c}}(2)=0.8$ and $T_{\mathrm{c}}(3)=1.0$, so that the 1 -st layer is the activated one and the 2 -nd and the 3 -rd layers are frozen. We notice that the shifting speed of peak position of $P_{n}\left(E_{n}, t\right)$ in the frozen layers is slower than that in the activated layer. In fact, it can be proved analytically that in the case of 
$t \gg 1$ and $T_{\mathrm{c}}(n-1)<T<T_{\mathrm{c}}(n)$, the energy distribution $P_{n}\left(E_{n}, t\right)$ satisfies the scaling,

$$
\begin{aligned}
P_{k}\left(E_{k}, t\right)=\bar{P}_{k}\left(E_{k}-R_{k} T \log t\right) & (k \geq n), \\
R_{k} & \equiv\left\{\begin{array}{cc}
1 & (k=n), \\
\prod_{l=n}^{k-1} \frac{T}{T_{\mathrm{c}}(l)} & (k>n) .
\end{array}\right.
\end{aligned}
$$

The validity is clear from the scaling plots shown in the insets of Fig. 6. The scaling implies that the age of the $k$-th $(k>n)$ frozen layer at $t$ is nearly equal to that of the activated layer at $t^{R_{k}}$, if age of each layer is measured by the peak position of the energy distribution. This frozen property becomes very important at experimental time scale $t_{\text {exp }}$, which is considered to be $10^{13} \leq t_{\exp } \leq 10^{18}$ in ordinary spin glasses in unit of the microscopic time of the system.

\section{Acknowledgment}

The numerical simulations were made on an Origin 2000 at Division of Physics, Graduate school of Science, Hokkaido University.

1) P. Svedlindh, P. Granberg, P. Nordblad, L. Lundgren and H. S. Chen: Phys. Rev. B 35 (1987) 268.

2) L. Lundgren, P. Svedlindh and O. Beckman: Phys. Rev. B 26 (1982) 3990.

3) P. Granberg, L. Sandlund, P. Nordblad, P. Svedlindh and L. Lundgren: Phys. Rev. B 38 (1988) 7097.

4) P. Nordblad and P. Svendlidh: in Spin-glasses and random fields, edited by A. P. Young, (World Scientific, Singapore, 1997); cond-mat/9810314.

5) Ph. Refreggier, E. Vincent, J. Hammann and M. Ocio: J. Phys. (Paris) 48 (1987) 1533.

6) P. Granberg, L. Lundgren and P. Nordblad: J. Magn. Magn. Mater 92 (1990) 228.

7) F. Lefloch, J. Hammann, M. Ocio and E. Vincent: Europhys. Lett 18 (1992) 647.

8) E. Vincent, J. P. Bouchaud, J. Hammann and F. Lefloch: Phil. Mag. B 71 (1995) 489.

9) J. O. Andersson, J. Mattsson and P. Nordblad: Phys. Rev. B 48 (1993) 13977.

10) D. S. Fisher and D. A. Huse: Phys. Rev. Lett. 56 (1986) 1601.

11) A. J. Bray and M. A. Moore: Phys. Rev. Lett. 58 (1987) 57.

12) D. S. Fisher and D. A. Huse: Phys. Rev. B 38 (1988) 373.

13) G. J. M. Koper and H. J. Hilhorst: J. Phys. (Paris) 49 (1988) 429.

14) J. P. Bouchaud and D. S. Dean: J. Phys. I (France) 5 (1995) 265.

15) M. Sasaki and K. Nemoto: J. Phys. Soc. Jpn. 69 (2000) 3045.

16) B. Derrida: J. Phys. Lett. (France) 46 (1985) 401.

17) B. Derrida and E. Gardner: J. Phys. C 19 (1986) 2253.

18) M. Sasaki and K. Nemoto: J. Phys. Soc. Jpn. 69 (2000) 2283.

19) M. Sasaki and K. Nemoto: J. Phys. Soc. Jpn. 69 (2000) 2642.

20) K. Jonason, E. Vincent, J. Hammann, J. P. Bouchaud and P. Nordblad: Phys. Rev. Lett. 81 (1998) 3243.

21) L. Bellon, S. Ciliberto and C. Laroche: preprint cond-mat/9906162.

22) P. Doussineau, T. Lacerda-Aroso and A. Levelut: Europhys. Lett 46 (1999) 401.

23) E. Vincent, V. Dupuis, M. Alba. J. Hammann and J. P. Bouchaud: preprint cond-mat/9908030.

24) J. Hammann, E. Vincent, V. Dupuis, M. Alba, M. Ocio and J. P. Bouchaud: preprint cond-mat/9911269. 
25) K. Nemoto: J. Phys. A 21 (1988) L287.

26) L. Bellon, S. Ciliberto and C. Laroche: preprint cond-mat/9905160.

27) R. L. Leheny and S. R. Nagel: Phys. Rev. B 57 (1998) 5154.

28) F. Alberich-Kious, J. P. Bouchaud, L. F. Cugliandolo, P. Doussineau and A. Levelut: Phys. Rev. Lett. 81 (1998) 4987.

29) L. F. Cugliandolo and J. Kurchan: Phys. Rev. B 60 (1999) 922. 


\section{FIGURE CAPTIONS}

Fig. 1 Structure of the GREM with $L=2$ and $N=5$. The open circles represent accessible states of the system.

Fig. 2 Out-of-phase ac-susceptibility $\chi^{\prime \prime}$ measured in the GREM with $L=2, T_{\mathrm{c}}(1)=0.5$ and $T_{\mathrm{c}}(2)=1.0$. The amplitude and the period of the applying ac-field are 0.1 and $100 \tau_{0}$, respectively. The system is cooled (and reheated) at the rate of $2.0 \times 10^{-5} T / \tau_{0}$. In the case of $\chi_{\text {wait }}^{\prime \prime}$ measurement, the cooling is intermitted at $T_{\text {wait }}=0.7$ for $1.0 \times 10^{5} \tau_{0}$. The solid line, open circles and full circles correspond to $\chi_{\text {ref }}^{\prime \prime}, \chi_{\text {wait }}^{\prime \prime}$ (cooling) and $\chi_{\text {wait }}^{\prime \prime}$ (reheating), respectively.

Fig. 3 Difference between $\chi_{\text {ref }}^{\prime \prime}$ and $\chi_{\text {wait }}^{\prime \prime}$ in the cooling and the reheating stages. The open circles and the full circles correspond to the cooling and the reheating data, respectively.

Fig. 4 Out-of-phase ac-susceptibility $\chi_{0}^{\prime \prime}$ and $\chi_{1}^{\prime \prime}$ evaluated from $\mathcal{M}_{0}$ and $\mathcal{M}_{1}$. The solid line, open circles and full circles correspond to $\chi_{\text {ref }}^{\prime \prime}, \chi_{\text {wait }}^{\prime \prime}$ (cooling) and $\chi_{\text {wait }}^{\prime \prime}$ (reheating), respectively.

Fig. 5 Effect of positive $T$-cycle in the Single-layer Random Energy model (the GREM with $L=1$ ) with $T_{\mathrm{c}}=1.0$. The ac-field with the peak amplitude 0.1 and the period $100 \tau_{0}$ is applied for the measurement of $\chi^{\prime \prime}$. After quenching from an infinitely high temperature, the system is kept at $T=0.5$. Then a positive temperature perturbation $\Delta T=0.35$ is applied at $t_{1}=4 \times 10^{3} \tau_{0}$ and is switched off at $t_{1}+t_{2}=1.4 \times 10^{4} \tau_{0}$. In the inset, $t_{2}$ part of data is omitted and $t_{1}$ and $t_{3}$ parts are connected for comparison with the unperturbed data (dotted line).

Fig. 6 Time evolution of $P_{n}\left(E_{n}, t\right)$ of the GREM with $L=3, T_{\mathrm{c}}(1)=0.6, T_{\mathrm{c}}(2)=0.8$ and $T_{\mathrm{c}}(3)=1.0$. After quenching from an infinitely high temperature, the system is kept at $T=0.45$ and $P_{n}\left(E_{n}, t\right)$ is measured at $t=10^{4.5}, 10^{5.0}, 10^{5.5}, \ldots, 10^{8} \tau_{0}$ (from left to right). In the inset, $P_{n}\left(E_{n}, t\right)$ is plotted as a function of $E_{n}-R_{n} T \log t$, where $R_{1}=1.0, R_{2}=0.75$ and $R_{3}=0.42$ (see text). 


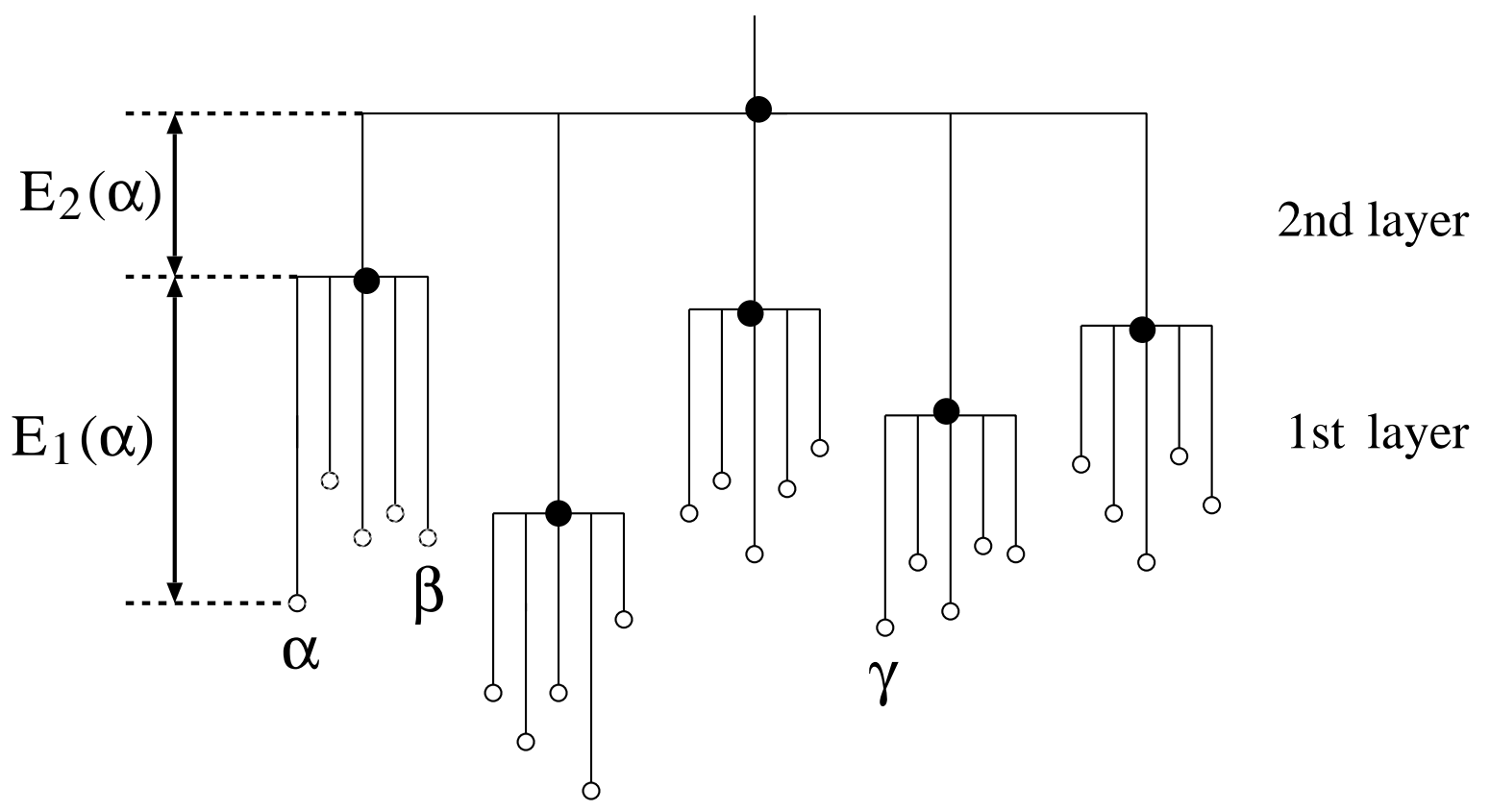

Fig.1 


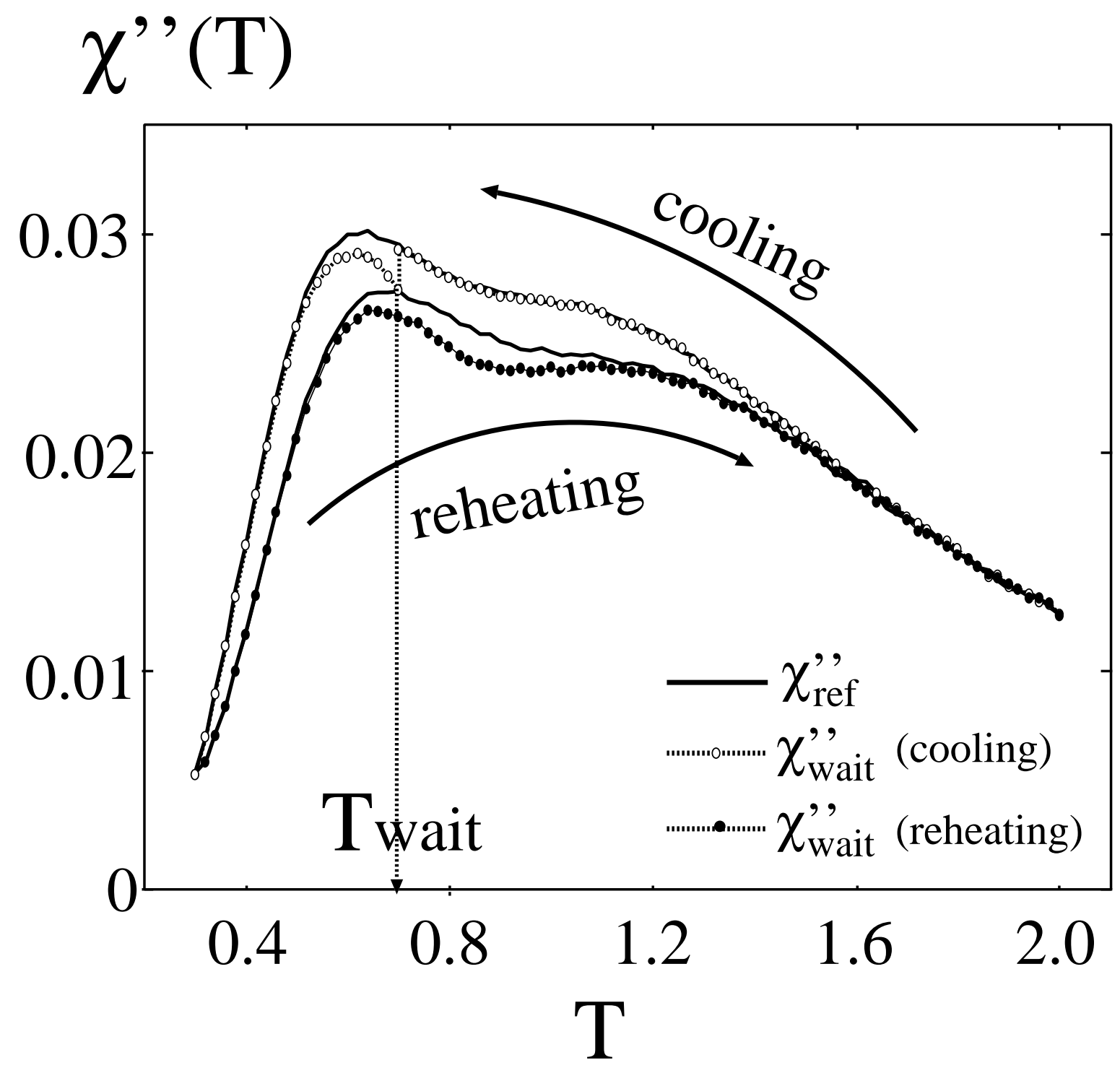

Fig.2 


$$
\chi_{\text {ref }}^{\prime \prime}-\chi_{\text {wait }}^{\prime \prime}
$$

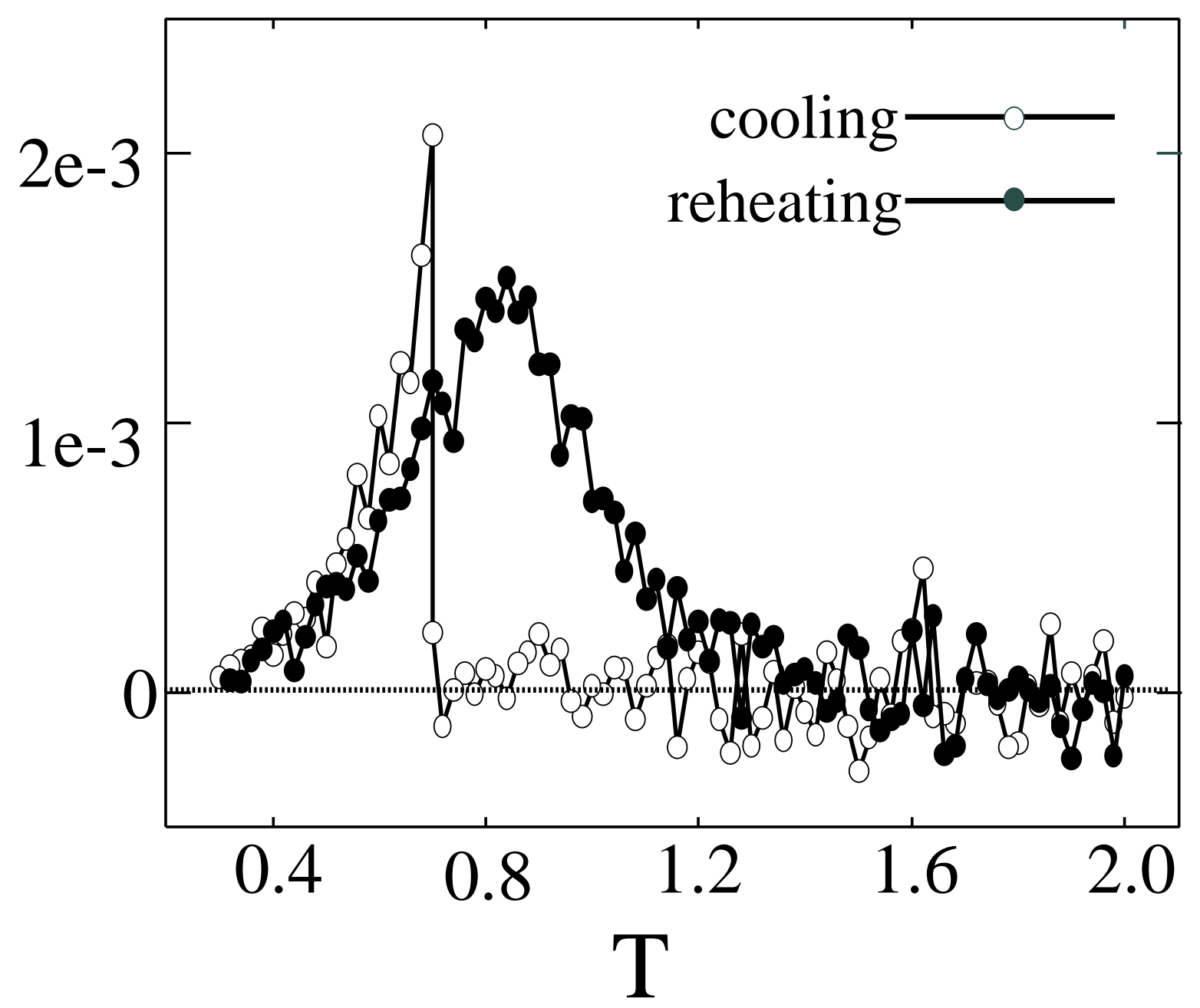

Fig.3 


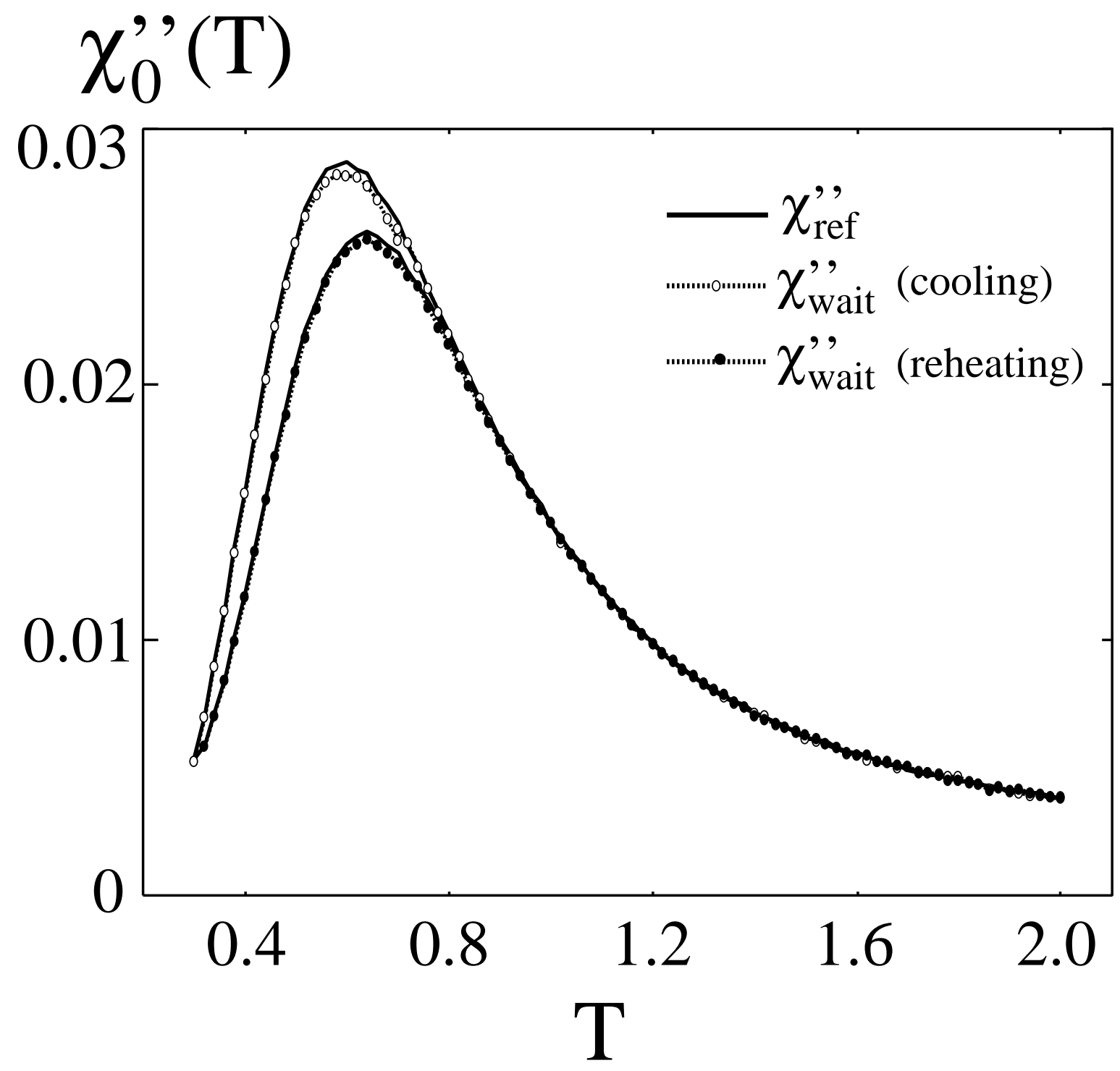

Fig.4(a) 


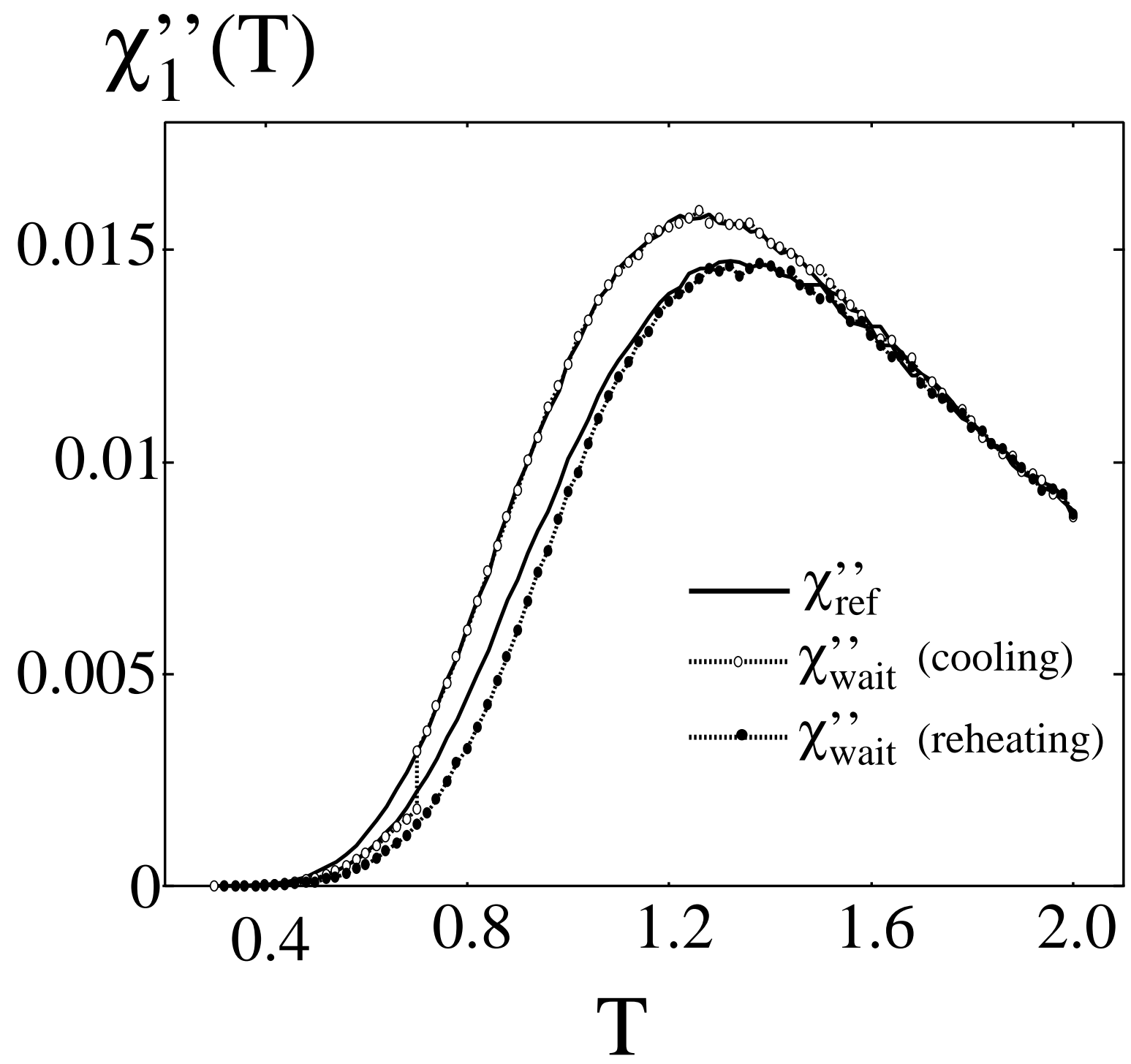

Fig.4(b) 


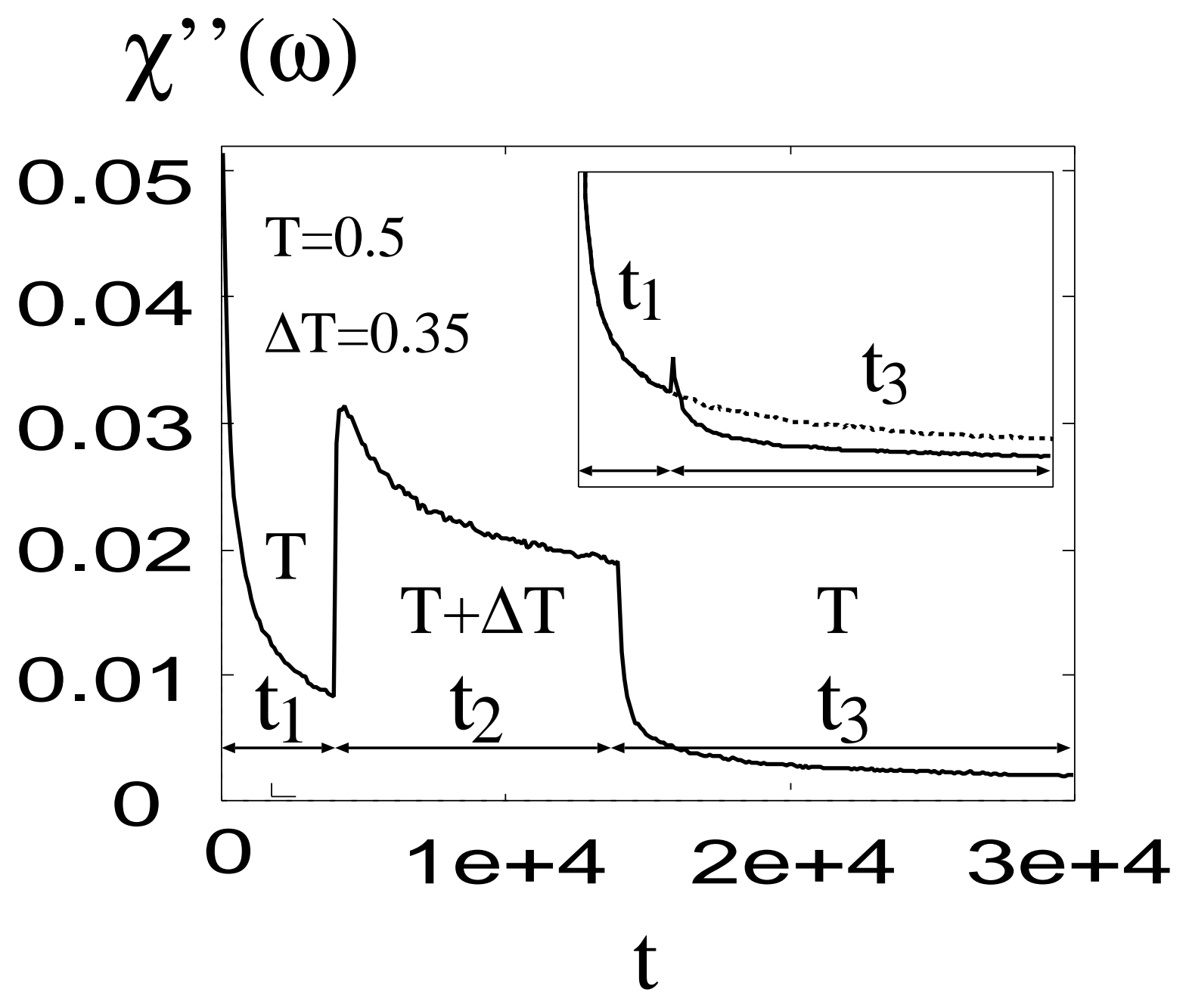

Fig.5 


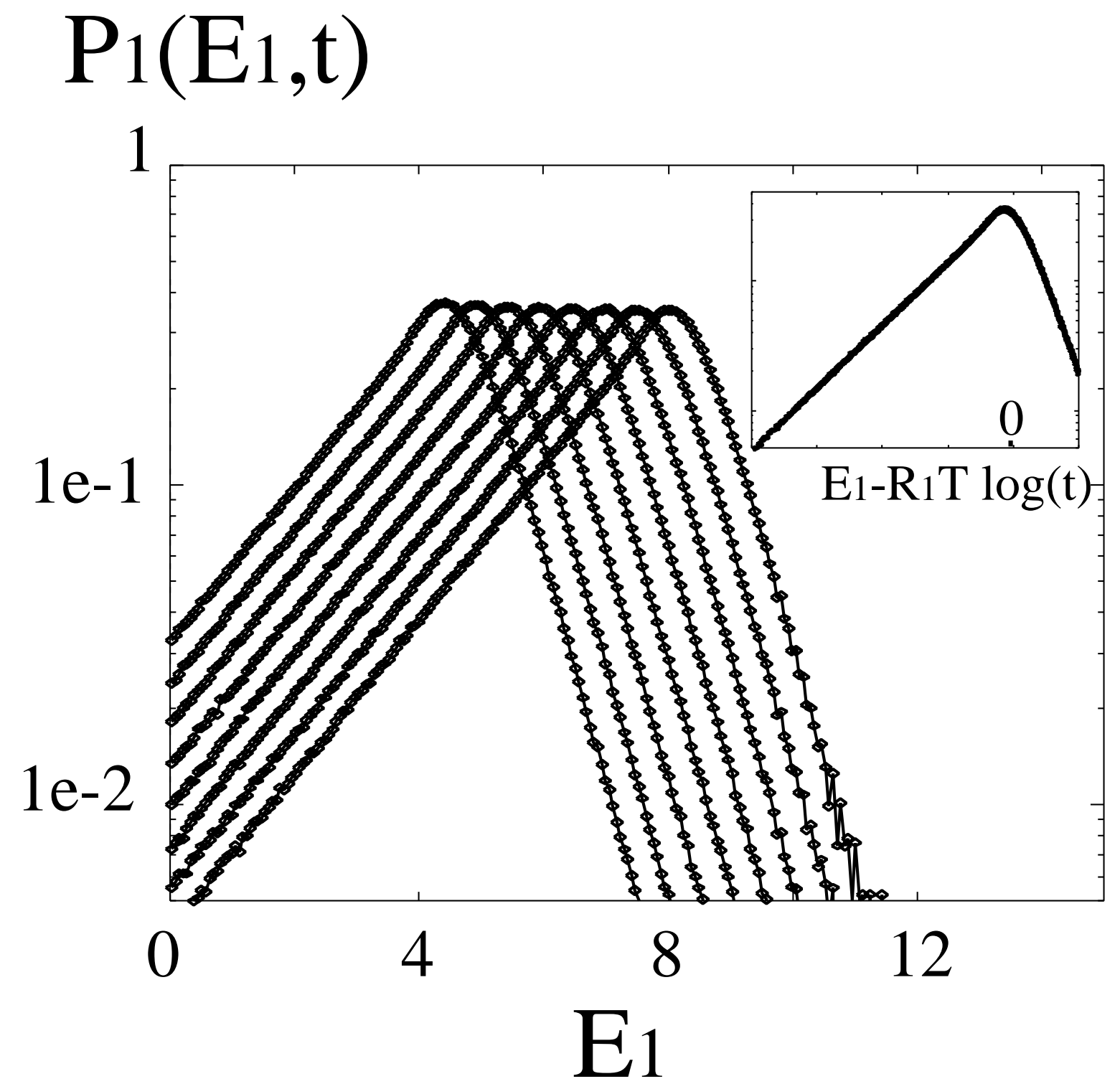

Fig.6(a) 


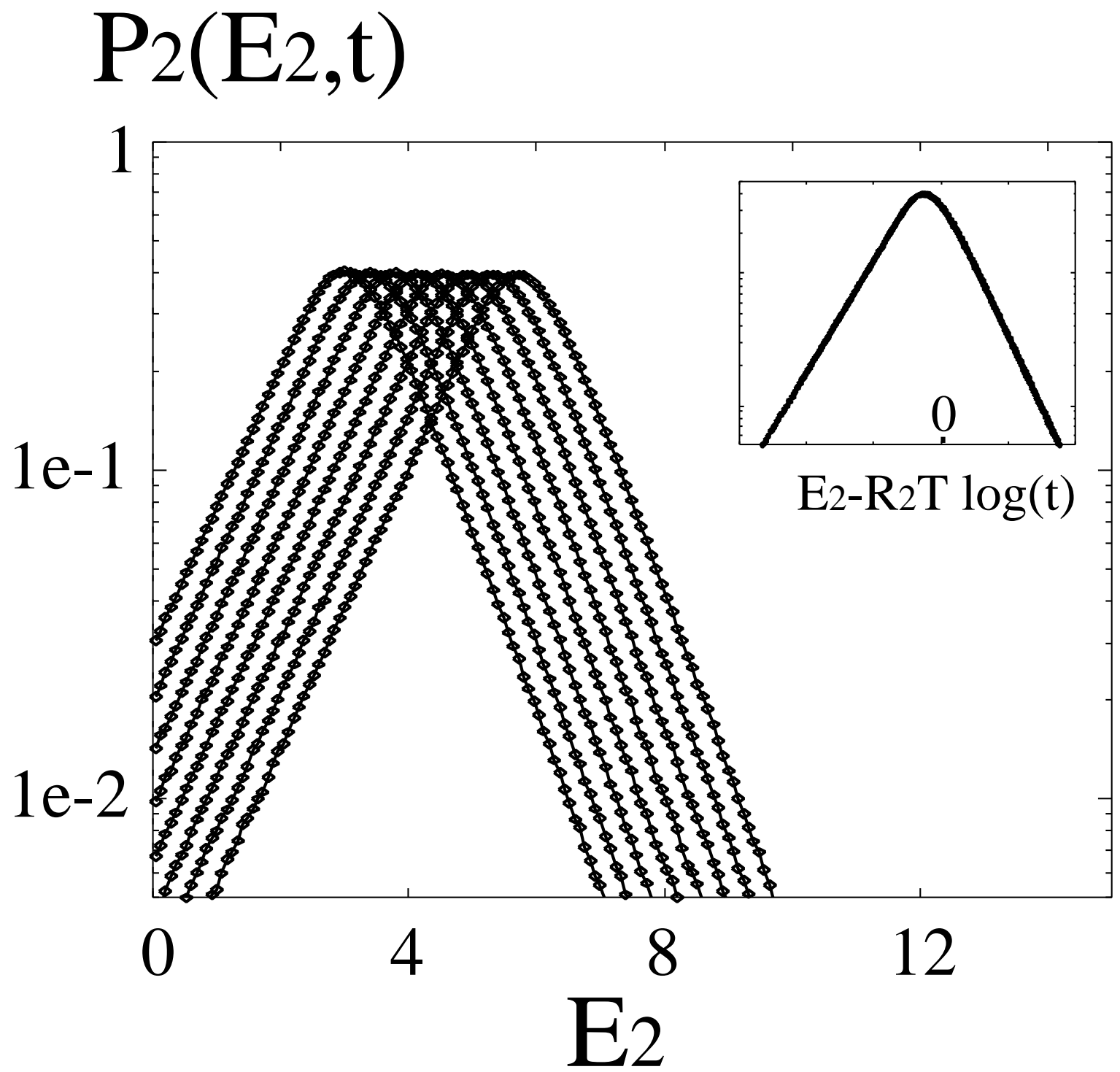

Fig.6(b) 


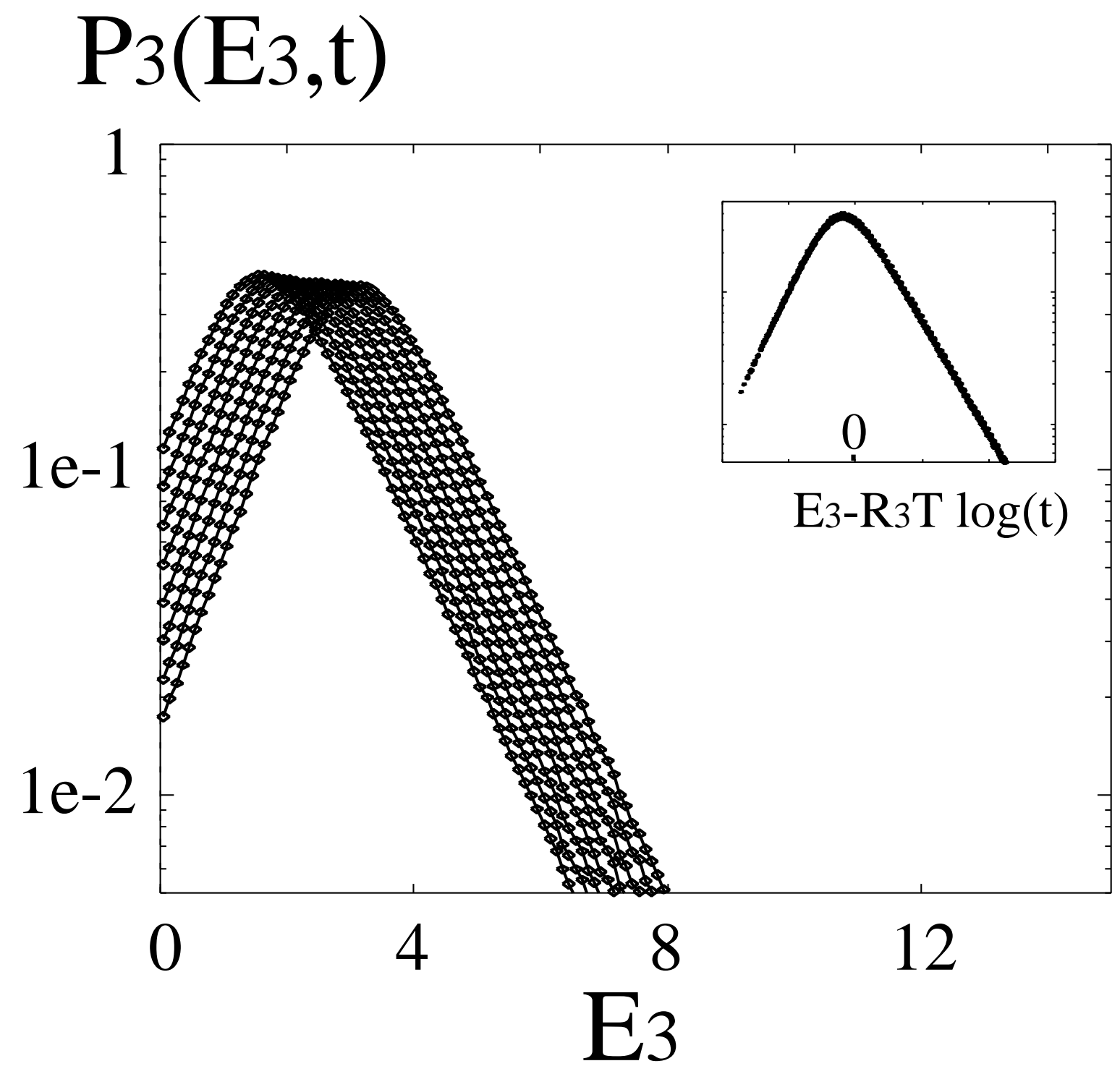

Fig.6(c) 